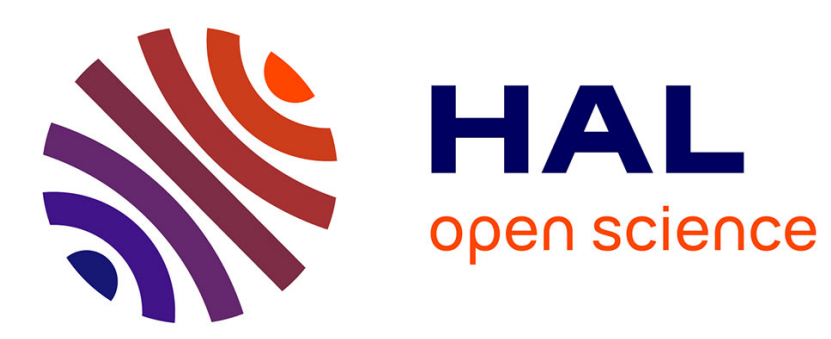

\title{
Integration of physical models in the ORQA framework for electric vehicle energy management
}

Borjan Tchakaloff, Sébastien Saudrais, Jean-Philippe Babau

\section{To cite this version:}

Borjan Tchakaloff, Sébastien Saudrais, Jean-Philippe Babau. Integration of physical models in the ORQA framework for electric vehicle energy management. Doctoral Symposium on Components and Architecture (WCOP), Jun 2014, Marcq-en-Baroeul, France. pp.7-12, 10.1145/2601328.2601332 . hal-01020135

\section{HAL Id: hal-01020135 \\ https://hal.univ-brest.fr/hal-01020135}

Submitted on 7 Jul 2014

HAL is a multi-disciplinary open access archive for the deposit and dissemination of scientific research documents, whether they are published or not. The documents may come from teaching and research institutions in France or abroad, or from public or private research centers.
L'archive ouverte pluridisciplinaire HAL, est destinée au dépôt et à la diffusion de documents scientifiques de niveau recherche, publiés ou non, émanant des établissements d'enseignement et de recherche français ou étrangers, des laboratoires publics ou privés. 


\section{Integration of Physical Models in the ORQA Framework for Electric Vehicle Energy Management}

\author{
Borjan Tchakaloff 1,2 \\ b.tchakaloff@estaca.fr \\ 1 CERIE, ESTACA \\ F-53000 Laval, France
}

\author{
Sébastien Saudrais ${ }^{1}$ \\ s.saudrais@estaca.fr
}

\author{
Jean-Philippe Babau ${ }^{2}$ \\ babau@univ-brest.fr
}

2 Univ. Bretagne Occidentale, UMR 6285, Lab-STICC

F-29200 Brest, France

\begin{abstract}
The energy management of electric vehicles is located in the storage system. It considers only the storage physical constituents and adapts the demand of the drive-chain technologies but is blind to other parts of the vehicle. Furthermore it has a very focused approach - to optimise the vehicle movement - and misses the driver's pragmatical needs. ORQA is a framework dedicated to the modelling of the vehicle consuming devices complementing the embedded systems models. It characterises each consuming device by its energetic needs and the quality levels it offers from a driver perspective. ORQA is realised by a system-level energy manager which looks for a driving strategy matching the driver needs and constraints. As the components used in ORQA are generic devices, the consumption of the devices is an approximation of their real consumptions. This paper presents the adaptation of the ORQA framework to have a closer estimation of the consuming devices. We propose the use of physical models issued from mechatronic systems for the main devices, drive-chain and energy storage, and discuss the benefits and constraints.
\end{abstract}

\section{Categories and Subject Descriptors}

D.2.11 [Software Engineering]: Domain-Specific Architectures; D.2.13 [Software Engineering]: Reusable Software-domain engineering, reuse models; J.7 [Computers in Other Systems]: Consumer Products - electric vehicle

\section{Keywords}

model driven architecture; energy consumption; mechatronic system; Quality of Service

\section{INTRODUCTION}

During the last years, the number of electric vehicles models have increased and their use is present in all the main countries. The main problem against this type of propulsion is the short autonomy provided, hence certain reluc-

Permission to make digital or hard copies of all or part of this work for personal or classroom use is granted without fee provided that copies are not made or distributed for profit or commercial advantage and that copies bear this notice and the full citation on the first page. Copyrights for components of this work owned by others than the author(s) must be honored. Abstracting with credit is permitted. To copy otherwise, or republish, to post on servers or to redistribute to lists, requires prior specific permission and/or a fee. Request permissions from permissions@acm.org. WCOP'14, June 30-July 4, 2014, Marcq-en-Baroeul, France.

Copyright is held by the owner/author(s). Publication rights licensed to ACM. ACM 978-1-4503-2593-6/14/06 ...\$15.00.

http://dx.doi.org/10.1145/2601328.2601332. tance about their usage (commonly known as range anxiety). Current vehicles can handle a few hundred kilometres. One way to deal with the embedded energy issue of the electric vehicles is through software. An energy management system (EMS) is a high level software which monitors and manages an environment through specific-purposed components. EMS are commonly used in (full- and hybrid-) electrical vehicles, though they mainly manage only the engine and ignore the end-user provided Quality of Service (QoS). To provide an efficient energy management and to take into account the user-related QoS, an electric vehicle EMS has to consider all the devices and the user expectations. Several works allow for complete analysis and validation of the software embedded in vehicles, such as EAST-ADL [4]. Though they have a systemic approach, they lack the energy dimension required to provide an EMS.

In [12], the ORQA framework is presented to tackle the global energy management while providing a QoS as good as possible. In ORQA, each embedded device is represented by a component and is characterised at design phase by its energy consumption(s) and its quality(ies), if applicable. The framework offers to realise a components architecture, in AUTOSAR [6] (an automotive component framework), which will elaborate on-line a solution to achieve the driver request (reach a destination) while providing the best possible vehicle QoS. The ORQA principle is to limit by software the devices and engine usage. In the previous works, consumption models of the drive-chain and the battery were simple and generic in order to validate the ORQA framework for any kind of electric vehicles. From an electric vehicle to another one, the characteristics of the different devices change. The use of models from the mechatronic domain offers models closer to the real devices.

In this paper, we show that accurate and specific models offer more precise estimation of the energy consumption. Moreover, the use of physical models offers also the possibility to apply ORQA to hybrid vehicles. The paper presents the physical models that can be introduced in ORQA and the corresponding constraints, and illustrates them on some case studies.

The rest of the paper is structured as follows. Section 2 presents the ORQA framework and its operation. Section 3 details the evolution of the current models to the physical models of the drive-chain and the battery. Section 4 discusses about the modification of the implementation of ORQA. Section 5 assesses the interest of a physical modelling. Finally, Section 6 concludes. 


\section{ORQA APPROACH}

ORQA is a framework to set up an energy management system for electrical vehicles. It is based on models of the devices energy consumption and models of user-oriented Quality of Service. It provides the energy and quality knowledge to the embedded systems. By the mean of an energy manager, ORQA assures the driver of a solution trip and of an on-line control of the devices based on her preferences.

\subsection{ORQA methodology}

At design stage, the designers create the energy and QoS models of the vehicle devices. The framework offers a library of predefined models to help them, comprising the engine and several devices such as the lighting system, the climate control unit and the entertainment system. An energy manager implementing these models is generated to take place in the embedded systems of the vehicle. It connects to the existing software and provides them with energy and quality knowledge. At run-time, the energy manager handles the driver request to find a solution trip. The inputs and the outputs of ORQA are illustrated in Figure 1. We now describe the framework operation in five steps.

1. The driver defines a destination point and its preferences. Driver preferences cover embedded devices ordering and consumption policy with possible constraints over trip duration and consumption. These definitions are later used to compute and select the best possible solution.

2. The possible routes are retrieved or generated from the system (e.g. from the GPS unit). Each route is refined to provide different driving conditions.

3. Each solution is evaluated, that is its energy consumption, trip duration, and global quality are computed. A solution is composed of a route and a combination of the devices operating states.

4. The solutions are passed through scoring functions depending on the driver preferences. Driver-defined constraints are used to discard undesirable driving strategies. The driver is then informed of the higher ranked solution as the best possible match.

5. Finally, the embedded energy manager is used to control the devices on-line throughout the trip. It controls the devices behaviour following the proposed strategy by the mean of brokers.

\subsection{Modelling the devices}

The vehicle devices are controlled by and supply information to the embedded systems (ES). The ES models already

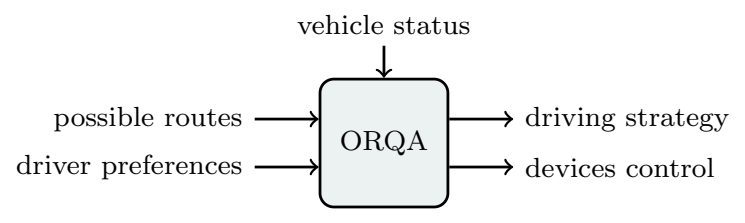

Figure 1: Overview of the ORQA Energy Management System. contain the vehicle devices. ORQA proposes to characterise both energy and quality of these devices in a specific model. It integrates them back in the existing ES models such as to keep the compatibility with the original meta-model.

A device is modelled as a software component, it offers a service. A service can be realised through different states, each with a defined quality. The physical realisation of a service consumes some energy. So we say that a device has one or more operating states realising a service, and each state is characterised by an energy requirement and a quality of service. As the devices are already modelled in the system to offer their services, their operating state are also accessible. ORQA extracts the devices and their operating states in its specific model. It allows the designers to characterise their energy requirements and quality levels. The energy requirement of an operating state is modelled by its required power. By multiplying this power to the usage duration of the device, we obtain the device energy consumption. The quality level of an operating state is a qualitative percentage of the given service $-100 \%$ is the best QoS possible.

\subsection{Providing a solution}

A request from the driver triggers the solution searching process of the energy manager. It evaluates the possible routes combined with the devices different uses. Each route retrieved from the system is refined into variations to represent different driving conditions. In this way each solution is composed of three elements: i) a route to destination, ii) a combination of devices operating states, and iii) the evaluation results (duration, energy consumption, and quality). The operating states combination of a solution is the optimal state in which the driver uses the devices according to their consumptions and qualities. It directly depends on the driver preferences and on the available energy during the journey. Scoring functions evaluate each solution results so that minimum (duration, consumption) or maximum (quality) results obtain the best score. The results scores are combined into the solution score according to weights defined by the driver preferences. The best solution is the one with the higher score; it becomes the driving strategy proposed by ORQA to the driver.

The driving strategy is computed before the effective vehicle departure. It is a prevision of the journey and by nature a journey has uncertainties such as the exact traffic flow and the driver behaviour.

\section{PHYSICAL MODELS}

In order to have a more accurate estimation, the models can be replaced by physical models. These new models will be specific for a given vehicle where the engine and the storage have been designed specifically so the associated models will vary between the different vehicles. The physical models concern the drive-chain, the energy storage and specificities of the hybrid vehicles case.

\subsection{The drive-chain}

The drive-chain of a vehicle is what makes it move, it goes from the engine to the wheels. The engine is the main consuming device of an electric vehicle. The engine of a simulated urban vehicle ${ }^{1}$ consumes about $57 \%, 79 \%$, and $93 \%$ of

\footnotetext{
${ }^{1}$ The simulated vehicle is described in [12], we assume a constant accessory load of $1500 \mathrm{~W}$.
} 


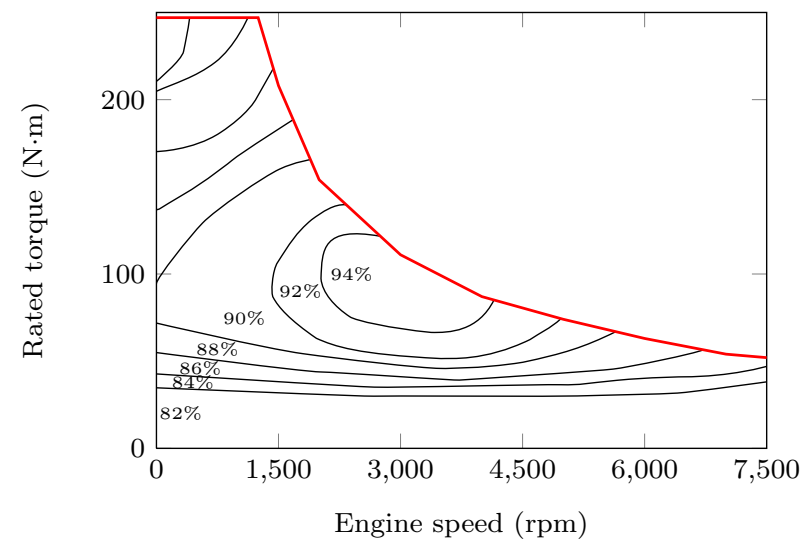

Figure 2: Engine efficiency based on engine speed and rated torque.

the total energy consumption in the Common Artemis Driving Cycles (CADC) [2] (respectively urban, rural, and motorway). We define the whole drive-chain required power as a function of the vehicle velocity, acceleration, and the road slope. This function is based on the well-known dynamic equations of an electric vehicle [8] and estimates the vehicle characteristics as constants. The engine required power is strictly negative when it is used for braking (i.e. regenerative braking that allows to charge the battery) and positive otherwise. This model is encapsulated in the component corresponding to the drive-chain in ORQA.

The vehicle dynamic equations are a simple and fast way to compute the engine required power to drive the vehicle. Yet an electric engine has dynamic characteristics (such as maximal torque and efficiency) that fluctuate along its speed and affect its power requirement. The current ORQA approach is to assume a constant efficiency for each engine mode (motor to drive the vehicle or generator when braking). The use of a physical model relying on an efficiency map offers a better estimation. It interpolates the engine efficiency for a given velocity and torque couple that can be deduced from the vehicle acceleration and velocity on a given road slope. Figure 2 shows an example of an engine efficiency map based on the engine speed and rated torque of a 30 kilowatts engine. For example, when the engine delivers a torque of $10 \mathrm{~N} \cdot \mathrm{m}$ (no matter its speed) its efficiency is of $82 \%$, meaning that at the end of the drive-chain $18 \%$ of the energy is lost. On the contrary, at a constant speed of $3000 \mathrm{rpm}$ and torque of $100 \mathrm{~N} \cdot \mathrm{m}$, only $6 \%$ of the consumed energy is lost. The physical model is able to give a more precise consumption based on more parameters and specific to the vehicle: rated torque and engine speed. During the solution selection, the candidate solutions with low efficiencies should be ignored and the high efficiency ones preferred. The actual overall efficiency of a driving strategy can be mixed in the engine QoS so that it is taken into account at the selection phase.

The design phase requires a specific development for each engine and for the corresponding vehicle. The knowledge of the mechatronic behaviour of the engine is needed to model and validate the efficiency map with experimental results. The replacement of the generic model by the physical model is done in ORQA by simply replacing the generic engine component by the specific vehicle's component.

\subsection{The energy storage}

The energy storage system - simplified by "the battery" in the rest of the paper - is ignored by the current version of ORQA apart from the remaining energy level. The component responsible of the battery just provides this level. This modelling does not take into account the physical response of batteries to the need of power. There are many storage technologies - such as batteries and fuel-cells - with different characteristics (maximal stored energy, maximal current, weight, and cost). Several works $[7,3,1,9]$ study the possibility of coupling different storage technologies to improve the system operation. Some types of storage are better to respond to instant demands of power while other have better results for long-term demands. The battery management system (BMS) must comply with the underlying storage technology and its characteristics, so it is calibrated specifically for a vehicle. From the ORQA point of view, the knowledge of the different elements of the battery permits to optimize a trip, not only on the vehicle consumption but also on how the energy is consumed.

\subsection{Hybrid vehicle}

An hybrid vehicle has an electric and a internal combustion engine (ICE) with the corresponding energy storage. The electric engine is used during urban phases and so the estimation solution has to only evaluate these phases but has to know the whole route to anticipate it. The model has to include the recharge phase of the battery with the modeling of a specific kinetic energy recovery system, KERS [10], which differs from a full electric vehicle. The ORQA methodology needs some adaptation to separate the part of the route operated by the ICE and the part operated by the electric engine. During the thermic phase, an estimation of the recharge of the battery has to be modelled to improve the estimation of the level of energy left. Depending on the hybrid type, the recharge is realise through the ICE and/or through regenerative braking.

\section{IMPLEMENTATION}

A prototype of the ORQA framework is implemented for Autosar applications. The consumption models are introduced in the Autosar components with model transformations. The integration of the physical models into ORQA does not change the prototype interface but may change its execution time and its memory load.

\subsection{Current implementation}

The first phase of ORQA occurs at conception at the embedded systems modelling. Though ORQA carries general concepts, our prototype is implemented to run on an AUTOSAR compliant system. AUTOSAR is a methodology to model the embedded systems widely spread in the automotive world. It stands on the software components paradigm and allows the reuse and share of functionalities through encapsulation. So our prototype actually relies on Autosar models of the embedded systems. The vehicle devices characterisation is realised in a dedicated model. It breaks down to three phases: 1) the devices are extracted from the available models, 2) they are characterised in a dedicated model, and 3) the initial models are enhanced with the characterisation model. The Figure 3 illustrates the three phases of the AUTOSAR transformation. 


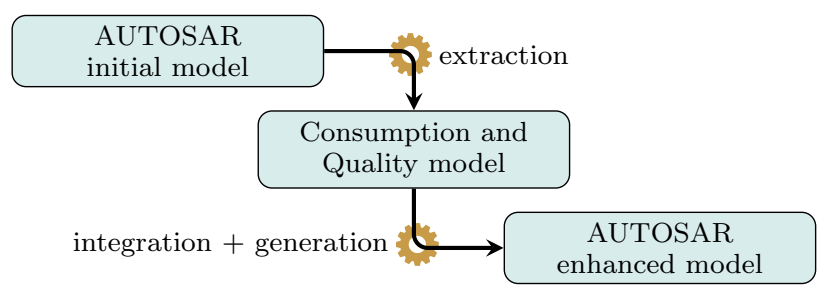

Figure 3: The AUTOSAR models transformations, from an initial model to an energy- and qualityaware model.

A first model transformation browses the initial models to extract the different devices. The energetic and qualitative characterisation model fits a specific Ecore meta-model [11]. The integration back in the initial models stays compatible with their interfaces, the existing components are not modified. So a second model transformation creates the new components (the Energy Manager, a broker per controlled device) and assures the good components linkage. Finally, a third model transformation generates the adequate implementation of the new components. In our prototype oriented Autosar, these are ANSI-C files.

\subsection{Physical models integration}

The integration of the physical models into ORQA does not have any impact on the transformation process from the initial AutosAR components to the energy-aware components. The models are integrated inside a specific component (the Energy Manager, EM) and communicate with the existing components by their original interfaces. The difference between a generic model and a physical model of an engine for an AUTOSAR application occurs during the second phase of the transformation: the consumption and quality modelling deviates accordingly. This model is then encapsulated inside the EM component.

\section{APPLICATION}

We want to assess the interest of a more detailed consumption model for ORQA. We take as illustration two engine models in ORQA, one theoretical and one physical, and compare their consumption to the engine consumption of a simulated electric vehicle. The simulation has the same engine characteristics that the two models. The difference lies in that the simulation follows a route trip based on the vehicle capacities, the simulated vehicle is driven in a closedloop to follow the route trip velocity reference. The ORQA evaluation uses a faster algorithm that assumes a static acceleration with three pre-defined values (negative, null or positive). We assume the simulation to have the more accurate results, so we compare both models to the simulation results. However, it should be noted that the models used in the simulation are not suitable for an on-line EMS as they have an important computational burden.

\section{Theoretical modelling of the engine.}

We make use of the engine model currently proposed by ORQA. It relies on the vehicle dynamics equations to represent the theoretical consumption of the engine. The equation is a function of the vehicle velocity and acceleration, and the road slope. The engine is assumed to have a constant efficiency in motor mode and another one in generator mode.

\section{Physical modelling of the engine.}

This more detailed model is defined with the engine efficiency map presented earlier (Figure 2). The engine consumption is the same than in the theoretical model except for the engine efficiency. It is not constant any more but is interpolated from the efficiency map. Also, as the presented efficiency map is about the motor, we assume an equivalent map in generator mode. Here, to use the physical modelling, we just replace the engine energy characterisation by the physical one. Then, the energy manager components are re-generated to take into account the model update.

\section{Discussion.}

Table 1 lists the engine consumptions of three generated routes for the simulation and the two engine models. These examples illustrates that, compared to the simulation, the physical model brings more accurate results than the theoretical model does. Respectively by $3.9,0.8$ and 4.5 points for the urban, rural and motorway routes.

These preliminary results show the interest for more detailed consumption models. With more accurate evaluations of the possible routes and their variations, ORQA can propose better solutions. Typically, this means that the best solutions use the engine optimal operation to provide a better Quality of Service.

The computation and memory overload are not detailed here. The execution time are not yet monitored as it requires special tooling. We can assume that the physical models require more time to evaluate because they are more complex. However, the memory overload can be statically measured as no dynamic allocations are allowed in the AUTOSAR components. The prototype shows a low static overload (ROM) of a few kilobytes. On the other hand, run-time memory (RAM) suffers an important overload of around ten kilobytes with this example modelling. A typical controller unit on which runs an AUTOSAR application features between 512 and 1024 kilobytes of ROM and between 32 and 64 kilobytes of RAM. So the memory overload of physical models must be closely measured to match the target controller capacities. In the case of an excessive overload (processor or memory), the physical models are simplified with discrete versions to lower their complexity.

\section{CONCLUSION}

This paper presented possible extensions of the ORQA framework to take into account other type of vehicles, like hybrid vehicles, and to have a more accurate estimation of the trip solution by using physical models. The framework is ready to incorporate the new models by replacing the generic components by more precise components containing mechatronic information. The validation of the physical models needs more feedback of a real vehicle as these models need to be adapted to each type of vehicle. The inclusion of the physical models in the on-line implementation also need more experimentation as they are more complex than the generic ones. The solution computation should comply with the system constraints over execution time and memory load. 


\begin{tabular}{lrrrrr}
\hline \multirow{2}{*}{ Route environment } & Simulation & \multicolumn{2}{c}{ ORQA: theoretical model } & \multicolumn{2}{c}{ ORQA: physical model } \\
& consumption & consumption & error & consumption & error \\
\hline urban & $388 \mathrm{Wh}$ & $371 \mathrm{Wh}$ & $-4.4 \%$ & $390 \mathrm{Wh}$ & $+0.5 \%$ \\
rural & $1814 \mathrm{Wh}$ & $1762 \mathrm{Wh}$ & $-2.9 \%$ & $1852 \mathrm{Wh}$ & $+2.1 \%$ \\
motorway & $8062 \mathrm{Wh}$ & $7046 \mathrm{Wh}$ & $-12.6 \%$ & $7409 \mathrm{Wh}$ & $-8.1 \%$ \\
\hline
\end{tabular}

Table 1: Engine consumption in a simulated vehicle compared to the evaluated consumptions in ORQA on three generated routes.

Other extensions to the solution search can be done by looking from a higher system point of view. Adding information about smart-home or smart-grid [5] will permit to optimise the consumption in both way (discharging the battery or storing the energy).

\section{REFERENCES}

[1] A. Allegre, A. Bouscayrol, and R. Trigui. Influence of control strategies on battery/supercapacitor hybrid energy storage systems for traction applications. In Vehicle Power and Propulsion Conference, 2009. VPPC '09. IEEE, pages 213-220, Sept 2009.

[2] M. André. The ARTEMIS european driving cycles for measuring car pollutant emissions. Science of The Total Environment, 334-335:73-84, 2004.

[3] H. Dai, X. Zhang, X. Wei, Z. Sun, J. Wang, and F. Hu. Cell-bms validation with a hardware-in-the-loop simulation of lithium-ion battery cells for electric vehicles. International Journal of Electrical Power and Energy Systems, 52(0):174 - 184, 2013.

[4] V. Debruyne, F. Simonot-Lion, and Y. Trinquet. East-adl - an architecture description language. In P. Dissaux, M. Filali-Amine, P. Michel, and F. Vernadat, editors, Architecture Description Languages, volume 176 of IFIP The International Federation for Information Processing, pages 181-195. Springer US, 2005.

[5] R. Druilhe, M. Anne, J. Pulou, L. Duchien, and L. Seinturier. Energy-driven consolidation in digital home. In Proceedings of the 28th Annual ACM Symposium on Applied Computing, SAC'13, pages 1157-1162. ACM, 2013.
[6] S. Fürst, J. Mössinger, S. Bunzel, T. Weber, F. Kirschke-Biller, P. Heitkämper, G. Kinkelin, K. Nishikawa, and K. Lange. Autosar-a worldwide standard is on the road. In 14 th International VDI Congress Electronic Systems for Vehicles, Baden-Baden, 2009.

[7] L. Gao, R. Dougal, and S. Liu. Power enhancement of an actively controlled battery/ultracapacitor hybrid. Power Electronics, IEEE Transactions on, 20(1):236-243, Jan 2005.

[8] J. Larminie and J. Lowry. Electric Vehicle Technology Explained, 2nd Edition. John Wiley \& Sons, 2nd edition, 2012.

[9] T. Mesbahi, N. Rizoug, P. Bartholomeus, and P. Le Moigne. A new energy management strategy of a battery / supercapacitor hybrid energy storage system for electric vehicular applications. In 7th IET International Conference on Power Electronics, Machines and Drives, 2014.

[10] J. Moreno, M. E. Ortúzar, and L. Dixon. Energy-management system for a hybrid electric vehicle, using ultracapacitors and neural networks. Industrial Electronics, IEEE Transactions on, 53(2):614-623, 2006.

[11] D. Steinberg, F. Budinsky, M. Paternostro, and E. Merks. EMF: Eclipse Modeling Framework, 2nd Edition. Addison-Wesley Professional. Part of the Eclipse Series series., 2nd edition, Dec. 2008.

[12] B. Tchakaloff, S. Saudrais, and J.-P. Babau. ORQA: Modeling energy and quality of service within AUTOSAR models. In Proceedings of the 9th international ACM Sigsoft conference on Quality of software architectures, QoSA'13, pages 3-12. ACM, 2013. 